\title{
Prevalence of Mucopolysaccharidosis I in A Paediatric and Young Adult Population, Diagnosed with Carpal Tunnel Syndrome
}

\author{
Domingo Ly-Pen ${ }^{1}$, José-Luis Andreu ${ }^{2}$, Gema de Blas ${ }^{3}$, Alberto Sánchez-Olaso ${ }^{4}$, Jesús Jiménez San Emeterio ${ }^{5}$ \\ ${ }^{1}$ GP Consultant. Abbey House Medical Centre. Navan. Co Meath. C15 D290. Ireland. \\ ${ }^{2}$ Head of Rheumatology Department, University Hospital Puerta de Hierro Majadahonda, Madrid - 28222, Spain. \\ ${ }^{3}$ Clinical Head, Neurophysiology Department, University Hospital Ramón y Cajal, Madrid - 28034, Spain. \\ ${ }^{4}(\dagger)$ Consultant, Plastic Surgery Department, University Hospital Ramón y Cajal, Madrid - 28034, Spain. (†Deceased 2012) \\ ${ }^{5}$ Paediatric Consultant. Gandhi Health Centre, Madrid - 28017, Spain
}

Corresponding author: Domingo Ly Pen; Abbey House Medical Centre; Navan. Co Meath. C15 D290. Ireland; domingoly@gmail.com

Received 21 May 2020;

Accepted 18 June 2020;

Published 02 July 2020

\begin{abstract}
Introduction: Mucopolysaccharidosis type I (MPS-I: Hurler/Scheie's disease) is a rare disease with a wide spectrum of severity. This results in underdiagnosis and delayed diagnosis. Prognosis greatly depends upon early diagnosis. The availability of reliable diagnostic tests and the existence of enzyme replacement therapy and/or hematopoietic stem cell transplantation, make an early diagnosis of these patients extremely important. Carpal tunnel syndrome (CTS) is among one of the most common findings in MPS I, whilst CTS is very uncommon in infancy and young adulthood. Objective: To ascertain the prevalence of MPS-I among children and young adults ( $\leq 30$ years) diagnosed with CTS in our population. To get an early diagnosis of MPS-I patients. Material and Methods: This is a cross-sectional prospective study of a cohort of patients with an electromyogram-confirmed diagnosis of CTS. Our total population is over 600,000 people of an urban area of Madrid. We searched for children and young adults with suggestive symptoms of CTS. $\chi 2$ for categorical variables and Student " $t$ " analysis or Anova test for quantitative variables were used. Results: 12 patients were included, 10 females, range 8-28 years, mean 23, median 23.5. Two patients had a low $\alpha$-L-Iduronidase activity. Nonetheless, the results of confirmatory test of enzymatic activity of $\alpha$-L-Iduronidase in lymphocytes were negative in both cases. Conclusions: We did not find any MPS-I in our series of CTS in children and young adults. Larger series will be needed to ascertain if this approach is valid for early diagnosis of MPS-I in the paediatric population.
\end{abstract}

Keywords: Carpal tunnel syndrome; Mucopolysaccharidosis I; paediatric population; early diagnosis.

\section{Background}

In 1919 Dr Gertrud Hurler described Mucopolysaccharidosis type I (MPS-I) also known as Hurler syndrome, a rare, progressive, lifethreatening autosomal recessive condition. In 1962 Dr Harold Scheie described a similar syndrome in adults, but symptoms were milder than in Hurler syndrome. There was a wide range of progressively debilitating manifestations with ongoing disease progression. The severity of visceral manifestations include bone involvement, cardiopathies, coarsening facial features, hepatomegaly, corneal clouding, umbilical and inguinal hernias ${ }^{[1,2]}$. At that time it was designated as Mucopolysaccharidosis type $\mathrm{V}$, as it was thought to have a different aetiology from MPS-I. In 1972, Hurler and Scheie syndromes were both recognised to be due to a deficiency of the enzyme $\alpha$-L-iduronidase. 2 Historically MPSI had been named into three separate diseases according to the severity of the clinical presentation: Hurler syndrome (severe), Hurler-Scheie syndrome (intermediate) and Scheie syndrome (mild). Despite being a rare disease, more than 100 different alleles of the a-L-iduronidase gene have been described. All of them can cause MPS-I, representing a disease continuum from severe to attenuated forms. Patients with the most severe disease start with signs and symptoms in early childhood, affecting simultaneously several different systems (rheumatologic, orthopaedic, cardiac, ophthalmologic, etc.), usually with evident cognitive impairment.1 On the other hand, patients with attenuated disease will not only develop fewer signs and symptoms but also will start later, with no cognitive impairment. In addition, diagnosis will be much more difficult and they can remain undiagnosed for many years ${ }^{[1,3]}$.

MPS-I belongs to a group of inherited metabolic disorders, resulting in progressive lysosomal accumulation of glycosaminoglycans in cells and tissues ${ }^{[4]}$. Because MPS-I is a rare disease and has such a broad spectrum of signs and symptoms, it is 
under-diagnosed and that diagnosis is often delayed ${ }^{[5]}$. In fact, we lack accurate data that could assess the epidemiology of attenuated forms of MPS I ${ }^{[6]}$, especially in Spain, our working environment.

Prognosis of the disease greatly depends upon early diagnosis. These facts and the availability of a rapid, sensitive and reliable test on Dry Blood Spot Filter Paper (DBS FP) for identifying $\alpha$-L-Iduronidase deficiency ${ }^{[7]}$, and the existence of hematopoietic stem cell transplantation and/or an Enzymatic Replacement Treatment for the disease make an early diagnosis of these patients dramatically important ${ }^{[4,8]}$.

Carpal tunnel syndrome (CTS) has a high prevalence (about $2.7 \%$ ) in the general population ${ }^{[9]}$, but CTS is very uncommon in infancy and young adulthood ${ }^{[4,9]}$. The prevalence of CTS in MPS-I patients is much higher, Thomas et al published a $67 \%$ CTS prevalence in patients with Scheie syndrome ${ }^{[10]}$.

CTS is among one of the most well-known and common findings in MPS-I ${ }^{[4,9]}$. In paediatric age, CTS symptoms can differ from those in adulthood: ${ }^{[11,12]}$ joint stiffness, flexion contractures, claw hand, trigger fingers, thenar atrophy, poor hand function and impaired hand motor skills (writing). In older children and adults, classic nocturnal hand paraesthesia are predominant features.

\section{Objective}

To ascertain the prevalence of MPS I among children and young adults ( $\leq 30$ years old) diagnosed with CTS in our population. To get an early diagnosis of MPS I patients.

\section{Methods}

Study design. Our study was originally designed to include patients retrospectively and prospectively. Initially, searching in the "Ramón y Cajal" University Hospital database, for patients diagnosed with CTS under the age of 30 years. Secondly, looking for new patients with suggestive symptoms, as explained in "Background". In these patients, electromyogram was performed when indicated. This aspect could be considered a cross-sectional prospective study of a cohort of patients with an electromyogramconfirmed diagnosis of CTS.

This study was approved by the Ethics Committees at our centres (Study code: GANDHI-08/01), and all patients (or legal guardians) gave written informed consent before inclusion in study.

Study population. The 4th Area of Madrid's Health System has a total population of over 600.000 people, in an urban area of Madrid.

Looking for candidates to be enrolled in this study, one of the authors (DLP) went to each of the 29 health centres in our geographic area (4th Area of Madrid's Health System), explaining our protocol to all general practitioners (GPs). Whenever a GP considered a patient to have symptoms clinically suggestive of CTS, the GP gave patient or legal guardian a leaflet about how to contact us (Table 1).

\section{Dr DOMINGO LY PEN. “GANDHI” Health Centre, C/ Gandhi no 30}

- By telephone: 913042927. Please, book an appointment with Dr LY PEN for "CTS".

- By fax: 913046775, directed to Dr LY PEN. Patient must write own data: name, phone number, best contact hour.

\section{Table 1: Information leaflet given by patient's GP}

After the initial assessment by DLP (Table 2), our study was explained to patients (or legal guardians) and they were invited to participate. Written, informed consent was obtained for every patient (or legal guardian) before enrolment (Table 3).

\section{Case report form:}

PROTOCOL: $\quad$ GANDHI / $08-01$

PATIENT'S CODE: 08/01/_ _

Date of birth (dd/mm/yyyy): ${ }_{-} I_{-} I_{-} I_{-}$

SEX: M/F. AGE: _-

Referring Health Centre:

Name and code of patient's GP:

Main diagnostic:

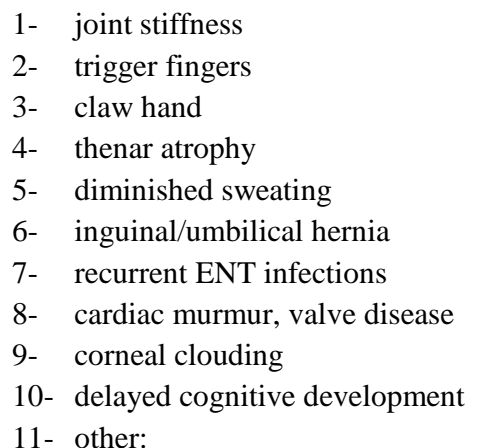

Presumptive diagnosis:

Past medical history:

Past surgical history:

Family history (disease and relationship): 


\section{Carpal tunnel syndrome:}

Date of diagnosis (dd/mm/yyyy): _ _ ${ }_{-} I_{-}-{ }_{-}$

Not diagnosed: $\quad \mathrm{x}$

Patient's dominant hand:

1- Right 2- Left

Affected side:

1- Unilateral 2-Bilateral

More affected hand:

1- Right

2- Left

Clinical symptoms:

1- Paraesthesias/daytime pain.

2- Paraesthesias/nocturnal pain.

3- Joint stiffness, claw hand.

4- Asymptomatic

5- Others:

Clinical signs:

1- Tinel

2- Phalen

3- Not valuable $1-2$

4- Thenar atrophy

5- Trophic changes in hand

6- Diminished sensitivity

\section{Other signs and symptoms:}

- RHEUMATOLOGIC:
1- Claw hand
2- Trigger fingers
3- Dysostosis
4- Others:

- GENERAL:
Inguinal/umbilical hernia Repeated ENT infections
Cardiac murmur. Valve disease.
Corneal clouding
Delayed cognitive retardation
Others:

- $\quad$ NOT CTS/MPS-I suspicion: Explain and send report to his/her family physician.

- $\quad$ LIKELY CTS/MPS-I suspicion:

- Require informed consent.

- Perform DBS test. Number of posting:

- $\quad$ Refer to clinical neurophysiology.

Date of appointment: ${ }_{--} I_{--} I_{----}$Hour: ${ }_{--} \cdot{ }_{--}$ Already done, result:

\section{$\underline{\text { Results of complementary studies: }}$}

ENG (median nerve): $\quad$ R- Right 1 - Normal

$$
\begin{array}{ll} 
& \text { 2- Mild } \\
& \text { 3- Moderate } \\
\text { 4- Severe } \\
\text { 1- Left } \\
\text { 2- Mild } \\
\text { 3- Moderate } \\
\text { 4- Severe }
\end{array}
$$

OTHER ENG:

cubital nerve:

radial nerve:

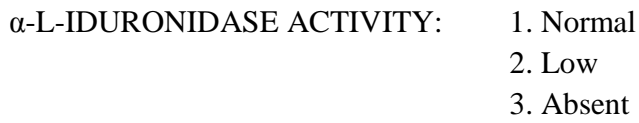




\section{Table 2: Case report form}

\section{Informed consent and authorisation form:}

Study for early detection of Mucopolysaccharidosis type I (MPS-I) in a paediatric and young adult population diagnosis with carpal tunnel syndrome in the $4^{\text {th }}$ Area of Madrid Health System.

Me: (name and surname)

As: - patient, - legal guardian of the child:

I have read this Informed Consent and Authorisation form,

I had had the opportunity to ask, and I had received answers to all questions. Regarding the study:

I understand that if I have any additional questions about my rights as a research participant, I may phone number 913042927

I agree to take part in this study as a research participant.

Signing this form, I confirm I am at least 18 years old and that I have received a copy of this Consent and Authorisation form.

You may withdraw your consent to participate in this study at any time.

You also have the right to cancel your permission to use and disclose further information collected about you at any time.

Your name will not be associated in any publication or presentation with the information collected about you or with the research findings from this study. Instead, the researcher(s) will use a study number or a pseudonym rather than your name. Your identifiable information will not be shared, unless required by law or you give additional written permission.

Permission granted on this date to use and disclose your information remains in effect indefinitely. By signing this form, you give permission for the use and disclosure of your information for purposes of this study at any time in the future.

$\begin{array}{lll}\text { Date: } & \text { Name of the parent or legal guardian } & \text { Signature } \\ \text { Date: } & \text { Name of investigator } & \text { Signature }\end{array}$

Table 3: Consent and authorisation form

When electromyogram confirmed clinical diagnosis of CTS, screening of the enzymatic deficit was done using the Dry Blood Spot Filter Paper test, to confirm or to rule out MPS-I (Figure 1). Patients with a positive test will be referred to the Rheumatology Department at Ramón y Cajal University Hospital (Figure 2).

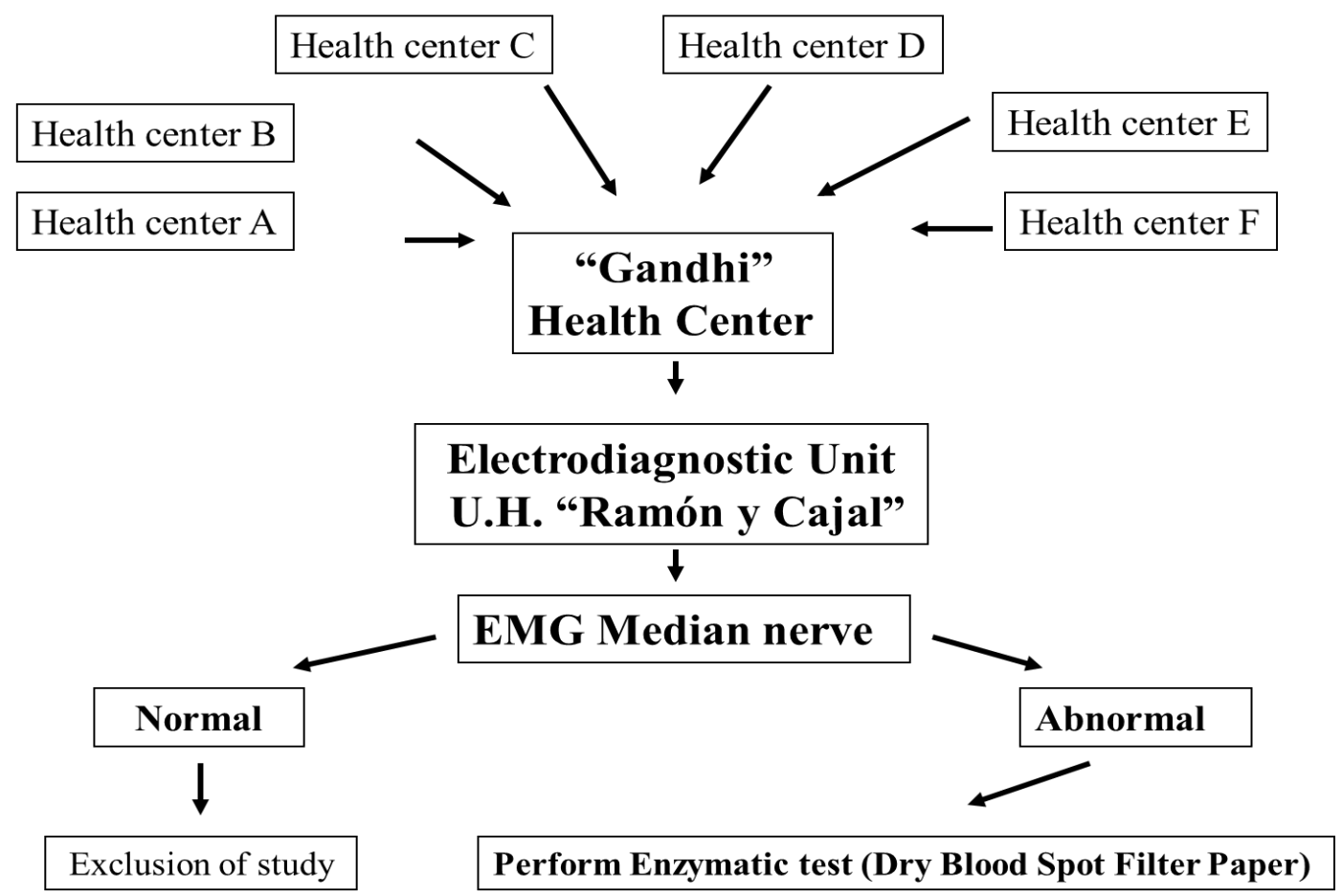

Figure 1: Diagram of patient's inclusion of our study 


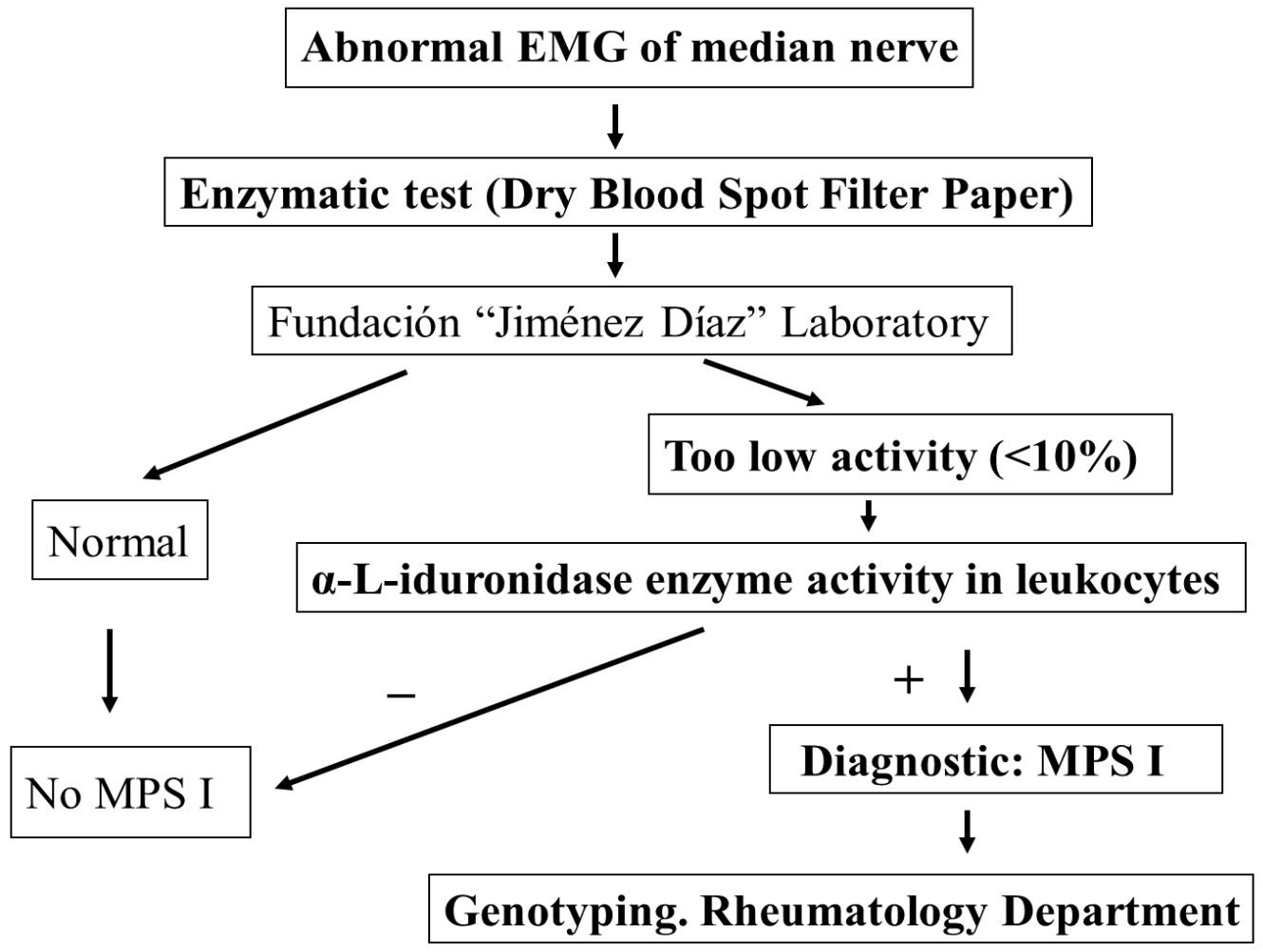

Figure 2: Diagram of patient's follow-up after CTS confirmation

Statistical analysis. A statistical description of the patients was done, summarising the quantitative variables with the mean and standard deviation, $\chi^{2}$ for categorical variables and Student "t" analysis or Anova test for quantitative variables were used.

We used the statistic program SPSS 15.0

\section{Results}

Unfortunately, due to both bureaucratic and technical problems, we were unable to contact patients retrospectively; in this way, only prospective new onset CTS patients could be included.

Twelve patients were enrolled, 10 females and 2 males; range 8 to 28 years, mean 23 years, median 23.5 years.

Two patients (a brother and sister) had a low $\alpha$-LIduronidase activity (5\% and 40\%) in the DBFP test. However, the results of confirmatory test of enzymatic activity of $\alpha$-LIduronidase in lymphocytes were negative in both cases.

\section{Discussion}

We were not able to diagnose any new case of MPS-I. We believe this is mainly due to the low number of cases included, as CTS is not a frequent disease in children.

The main problem enrolling the retrospective patients was the new Spanish Data Protection Act, which come into force when our protocol was started. In the last 30 years, in our reference hospital universitario "Ramón y Cajal", there could be about 150 patients fulfilling inclusion criteria.

It is beyond any doubt the crucial importance of the early diagnosis of Hurler-Scheie and Scheie patients. There have been many approaches to this early diagnosis for early treatment in order to avoid disease progression to irreversible life-threatening status. Scheie syndrome early diagnosis is quite challenging because patient's appearance can be completely normal. Furthermore, in some patients CTS may be the only clue to diagnosis in this group of patients ${ }^{[13]}$.
The only paper we were able to find in the literature, similar to ours, was from Normark et al ${ }^{[14]}$. In a similar study, they enrolled patients both prospectively and retrospectively. As in our study, they could not diagnose any new MPS-I, MPS-II, nor MPSVI patients. Despite their enrolment of a total of 425 CTS patients, only 20 of them were known to be diagnosed with CTS below the age of 18. Their inclusion criteria were below age 60, among others, but it is not specified how many were between 19 and 30 years old. They concluded that screening of MPS in adult patients with mono-symptomatic CTS was not indicated. In younger patients with CTS, they could not exclude this screening could be beneficial, as they included a small number of children.

More recently, Colon et al reported a novel selective screening program (FIND project), targeting children aged 0 to 16 years with clinical manifestations of MPS ${ }^{[15]}$. They used the fluorometric analysis of glycosaminoglycan levels, with paper impregnated with urine. This was applicable to all types of MPS (except type IX). This procedure can be sent through the post, being a simple and cost-effective method and may be a valuable first tier screening test for high-risk groups ${ }^{[15]}$.

Due to the clinical heterogeneity and rarity of MPS-I, theoretically the newborn screening should be the earliest way to identifying individuals MPS-I and abort the onset of irreversible clinical disease. Unfortunately, this screening is still in development.

In the meantime, we are afraid that only increasing physicians' suspicions and awareness of the MPS signs and symptoms will enable early, accurate diagnosis and treatment, improving patient outcomes ${ }^{[1]}$.

\section{Conclusions}


Likely screening for MPS in adult patients with mono-symptomatic CTS is not useful. In children and those below 18, the possibility that this screening could not be beneficial had not been excluded yet, as the only two available studies included a limited population of children ${ }^{[14,16]}$.

To diagnose the majority of MPS patients, either a more general approach (like newborn screening) or greater awareness of polysymptomatic suspicious patients is needed ${ }^{[14]}$.

\section{Acknowledgments}

The authors are indebted to Dr Joseph Hogg, partner at Abbey House Medical Centre, for his critical review, helpful comments and careful English revision.

\section{Declarations}

\section{Funding}

No funding available. All authors declare no support from any organisation for the submitted work.

\section{Conflicts of interest/Competing interests}

The authors declare that they have no conflict of interest. No benefits in any form had been received or will be received from a commercial party related directly or indirectly to the subject of this article.

\section{Ethics approval}

All procedures performed in studies involving human participants were in accordance with the ethical standards of the institutional research committee and with the 1964 Helsinki Declaration and its later amendments or comparable ethical standards. This study was approved by the Ethics Committee of the University Hospital Ramón y Cajal.

\section{Consent to participate}

All patients signed informed consent before enrolling the study. No individual's personal data is included

\section{Consent for publication}

Domingo Ly, the corresponding author, has the right to grant on behalf of all authors, and do grant on behalf of all authors, an exclusive licence, on a worldwide basis.

\section{Availability of data and material (data transparency)}

Please contact author for data requests.

\section{Authors' contributions}

DLP conceived the study, attended and reviewed the patients, collected data, conducted the literature review, wrote the paper and is the corresponding author. JLAS designed, coordinated the study, provided statistical consulting, analysed and interpreted the patient data, and helped in writing the paper. GdBB did the neurophysiological studies of all patients, assisted in the analysis of data and helped in writing the paper. ASO helped in study design and assisted in the analysis of data. JJSE assisted in the analysis of data and helped in writing the paper. All authors read and approved the final manuscript.

\section{References}

[1] Muenzer J. Overview of the mucopolysaccharidoses. Rheumatology 2011;50:4-12. doi:10.1093/rheumatology/ker394

[2] Bach G, Friedman R, Weissmann B, Neufeld EF. The defect in the Hurler and Scheie syndromes: deficiency of alfa-L-iduronidase. Proc Natl Acad Sci USA 1972;69:2048-51.

[3] Vijay S, Wraith JE. Clinical presentation and follow-up of patients with the attenuated phenotype of mucopolysaccharidosis type I. Acta Paediatr 2005;94:8727.

[4] Patel P, Antoniou G, Clark D, Ketteridge D, Williams N. Screening for Carpal Tunnel Syndrome in Patients With Mucopolysaccharidosis. J Child Neurol 2020;35(6):4107 088307382090448. doi:10.1177/0883073820904481

[5] D'Aco K, Underhill L, Rangachari L, Arn P, Cox GF, Giugliani R, et al. Diagnosis and treatment trends in mucopolysaccharidosis I: Findings from the MPS I registry. Eur J Pediatr 2012;171:911-9

[6] Beck M, Arn P, Giugliani R, Muenzer J, Okuyama T, Taylor $\mathbf{J}$ et al. The natural history of MPS I: Global perspectives from the MPS I Registry. Genet Med 2014; 16: 759-65

[7] Chamoles NA, Blanco MB, Gaggioli D, Casentini C. Hurler-like Phenotype: Enzymatic Diagnosis in Dried Blood Spot on Filter Paper. Clin Chem 2001; 47: 2098102.

[8] Van der Linden, MH, Kruyt, MC, Sakkers, RJ, De Koning, TJ, Oner, FC, Castelein, RM. Orthopaedic management of Hurler's disease after hematopoietic stem cell transplantation: a systematic review. J Inherit Metab Dis. 2011;34(3):657-69.

[9] Atroshi I, Gummesson C, Johnsson R, Ornstein E, Ranstam J, Rosén I. (1999). Prevalence of carpal tunnel syndrome in a general population. JAMA, 282, 153-8. https://jamanetwork.com/journals/jama/fullarticle/77426 3

[10] Thomas JA, Beck M, Clarke JT, Cox GF. Childhood onset of Scheie syndrome, the attenuated form of mucopolysaccharidosis I. J Inherit Metab Dis. 2010;33:421-7.

[11] Yuen A, Dowling G, Johnstone B, Kornberg A, Coombs C. Carpal tunnel syndrome in children with mucopolysaccharidoses. J Child Neurol. 2007;22(3):260-3.

[12] White, K, Kim, T, Neufeld, J. Clinical assessment and treatment of carpal tunnel syndrome in the mucopolysaccharidoses. J Pediatr Rehabil Med. 2010;3(1):57-62.

[13] Gökay S, Kardaş F, Kendirci M, Sözeri B. Arthropathylike findings and a carpal tunnel syndrome as the presenting features of Scheie syndrome: Three cases from the same family. Turkish J Pediatr 2018; 60: 344-7 DOI: 10.24953/turkjped.2018.03.020

[14] Nørmark, MB, Kjaer, N, Lund, AM. Prevalence of mucopolysaccharidosis types I, II, and VI in the pediatric and adult population with carpal tunnel syndrome (CTS). 
Retrospective and prospective analysis of patients treated for CTS. JIMD Rep. 2017;36:29-33. https://www.ncbi.nlm.nih.gov/pmc/articles/PMC5680292 /\#CR11

[15] Colón C, Alvarez JV, Castaño C, Gutierrez-Solana LG, Marquez AM, O'Callaghan $\mathrm{M}$ et al. A selective screening program for the early detection of mucopolysaccharidosis: Results of the FIND project - a 2-year follow-up study. Medicine (Baltimore). 2017 May; 96 (19): e6887. doi:
10.1097/MD.0000000000006887. PMID: 28489793; PMCID: $\quad$ PMC5428627. https://www.ncbi.nlm.nih.gov/pmc/articles/PMC5428627 I

[16] Ly-Pen D, Andreu J, de Blas G, Sanchez-Olaso A, Jiménez San-Emeterio J. Prevalence of Mucopolysaccharidosis I in a pediatric and young adult population diagnosed with carpal tunnel syndrome [abstract] Ann Rheum Dis. 2010;69(suppl 3):113 